Revue d'histoire de l'Amérique française

REVUE D.HISTOIRE DE L'AMÉRIQUE FRANÇAISE

\title{
Les principaux intervenants dans l'évolution du système hospitalier en Mauricie, 1889-1939
}

\section{François Guérard}

Volume 48, numéro 3, hiver 1995

URI : https://id.erudit.org/iderudit/305350ar

DOI : https://doi.org/10.7202/305350ar

Aller au sommaire du numéro

Éditeur(s)

Institut d'histoire de l'Amérique française

ISSN

0035-2357 (imprimé)

1492-1383 (numérique)

Découvrir la revue

Citer cet article

Guérard, F. (1995). Les principaux intervenants dans l'évolution du système hospitalier en Mauricie, 1889-1939. Revue d'histoire de l'Amérique française, 48(3), 375-401. https://doi.org/10.7202/305350ar
Résumé de l'article

Le système hospitalier au Québec durant la première moitié $\mathrm{du} \mathrm{XX}^{\mathrm{e}}$ siècle est souvent perçu comme relativement stationnaire du point de vue de l'organisation et des rapports de pouvoir entre les principaux intervenants. C'est là une image que nous avons voulu remettre en question, notamment en montrant que le clergé francophone, propriétaire ou gérant de la plupart des grands établissements, dut tenir compte non seulement des pressions des médecins desservants, dont le prestige et l'influence croissaient rapidement, mais aussi de l'entrée en scène dans ce champ d'activité de deux acteurs puissants : la grande entreprise et l'État. Afin d'atteindre cet objectif, nous avons effectué une étude de l'évolution des services hospitaliers dans deux villes québécoises, Trois-Rivières et Shawinigan.
Tous droits réservés @ Institut d'histoire de l'Amérique française, 1995
Ce document est protégé par la loi sur le droit d'auteur. L'utilisation des services d'Érudit (y compris la reproduction) est assujettie à sa politique d'utilisation que vous pouvez consulter en ligne.

https://apropos.erudit.org/fr/usagers/politique-dutilisation/ 


\title{
LES PRINCIPAUX INTERVENANTS DANS L'ÉVOLUTION DU SYSTÈME HOSPITALIER EN MAURICIE, 1889-1939 1
}

\author{
FRANÇOIS GUÉRARD \\ Centre interuniversitaire d'études québécoises \\ Université du Québec à Trois-Rivières
}

\section{RÉSUMÉ}

Le système hospitalier au Québec durant la première moitié du $\mathrm{XX}^{\mathrm{e}}$ siècle est souvent perçu comme relativement stationnaire du point de vue de l'organisation et des rapports de pouvoir entre les principaux intervenants. C'est là une image que nous avons voulu remettre en question, notamment en montrant que le clergé francophone, propriétaire ou gérant de la plupart des grands établissements, dut tenir compte non seulement des pressions des médecins desservants, dont le prestige et l'influence croissaient rapidement, mais aussi de l'entrée en scène dans ce champ d'activité de deux acteurs puissants: la grande entreprise et l'État. Afin d'atteindre cet objectif, nous avons effectué une étude de l'évolution des services hospitaliers dans deux villes québécoises, Trois-Rivières et Shawinigan.

\section{ABSTRACT}

The Quebec hospital system during the first half of the twentieth century is frequently seen as relatively stationary regarding its organization and the power relations between the main actors. The paper suggests that this perception must be reevaluated, since the French-speaking clergy, which owned or directed most of the principal hospitals, had to cope with the growing prestige and influence of the doctors, and with the arrival of two powerful players in this field of activity, largescale enterprise and the state. The argument is based upon a study of the evolution of hospital services in two Quebec cities, Trois-Rivières and Shawinigan, from 1889 to 1939.

1. La matière de cet article provient de la thèse de doctorat de l'auteur, La santé publique dans deux villes du Québec de 1887 à 1939. Trois-Rivières et Shawinigan, Université du Québec à Montréal, juillet 1993, 525 p., réalisée sous la direction de Paul-André Linteau et de Camille Limoges. Je tiens à remercier vivement Camille Limoges, Paul-André Linteau et France Normand dont les commentaires sur une version préliminaire de cet article ont été fort enrichissants.

RHAF, vol. $48, \mathrm{n}^{\circ} 3$, hiver 1995 
On a souvent dit du système hospitalier québécois francophone de la première moitié du $\mathrm{XX}^{\mathrm{e}}$ siècle que ses principales transformations avaient été guidées par une petite bourgeoisie traditionaliste maître des institutions politiques et réfractaire aux interventions de l'État dans le domaine de l'aide sociale. Dans cette optique, le clergé, propriétaire ou administrateur de la plupart des établissements, aurait exercé sur l'évolution des services un contrôle souvent presque sans partage, et serait, avec les gouvernements en place, plus ou moins responsable d'un retard du Québec dans la modernisation de ses services sociosanitaires. Pareil retard n'aurait été finalement balayé que lors des réformes des années 1960 et 1970.

Dans cet article, nous voulons remettre en question cette perception d'un système hospitalier presque immobile ${ }^{2}$, et le cadre interprétatif dans lequel elle s'insère. Pour ce faire, nous avons étudié l'évolution des services hospitaliers de la fin du XIX ${ }^{\mathrm{e}}$ siècle à 1939 dans deux villes du Québec, Trois-Rivières et Shawinigan. On le verra, il y eut dans le domaine hospitalier au Québec, à tout le moins en Mauricie, une plus grande diversité et des transformations bien plus profondes qu' on ne le croit généralement. Nous nous attacherons ainsi à démontrer qu'en plus des phénomènes de croissance et de médicalisation des hôpitaux déjà relevés par divers auteurs ${ }^{3}$, on assiste durant cette période d'un demi-siècle à une redéfinition du partage des pouvoirs dans ce secteur, notamment en raison des interventions de la grande entreprise à partir de la fin des années 1910, puis de l'État au début de la décennie $\mathbb{1 9 2 0}$.

À vrai dire, il n'existe pas d'étude consacrée spécifiquement au système hospitalier québécois durant la première moitié du $\mathrm{XX}^{\mathrm{e}}$ siècle. On trouve tout de même de nombreuses monographies d'établissements de soins qui, le plus souvent, présentent l'image d'un milieu consensuel au sein duquel médecins et communautés religieuses œu-

2. Un auteur comme Marc Renaud a ainsi écrit: «In contrast to the contemporary situation, the health sector in Quebec until the early 1960's seemed a most unchanging world with a static pattern of social relations and, few and limited state interventions. The main health and other social institutions were rooted into the concept of charity and, ideologically at least, they were still very much anchored to the rural parish.» The Political Economy of the Quebec State Interventions in Health: Reform or Revolution?, thèse de doctorat, University of Wisconsin, $1976,36$.

3. André Paradis et Denis Goulet relèvent par exemple que durant «les premières décennies du $X^{\mathrm{e}}$ siècle, les institutions hospitalières connaissent une croissance sans précédent», et que «la médicalisation des hôpitaux (...) s'accentue de façon remarquable». Voir Denis Goulet et André Paradis, Trois siècles d'histoire médicale au Québec. Chronologie des institutions et des pratiques (1639-1939) (Montréal, vlb éditeur, 1992), 40. 
vraient dans l'harmonie pour le bien-être de la population ${ }^{4}$. Plusieurs de ces ouvrages, anciens et récents, célèbrent une organisation traditionnelle des services reposant sur la charité privée et où le clergé occupait les postes de commande. Tout à l'opposé, depuis la Seconde Guerre mondiale, certains intellectuels ont dénoncé le statisme des institutions hospitalières de la première moitié du $\mathrm{XX}^{\mathrm{e}}$ siècle. Ainsi en est-il de ceux qui, à partir des années 1950 surtout, ont réclamé toute une série de réformes majeures où l'État deviendrait le principal pourvoyeur de services hospitaliers ${ }^{5}$. Quant aux auteurs d'inspiration marxiste, ils ont argué au cours des années 1970 et 1980 que le conservatisme de la petite bourgeoisie québécoise en matière sociosanitaire avait contribué à consolider la domination de la bourgeoisie sur les travailleurs ${ }^{6}$. Chez ceux qui ont vanté les mérites du système hospitalier québécois d'autrefois comme chez ceux qui l'ont dénigré, on songeait généralement peu à nuancer l'image qui s'était imposée

4. Voici une liste non exhaustive des monographies d'hôpitaux parues au Québec. En général, les ouvrages commémoratifs ou les plus anciens s'attachent surtout à mettre en valeur le travail des dirigeants et du personnel, tout en mettant parfois l'accent sur les progrès de la médecine hospitalière: Histoire de l'Hôtel-Dieu de Chicoutimi 1884-1934 (Chicoutimi, Imprimerie du progrès du Saguenay, 1934), 421 p.; Alain Gamelin, 1886-1986, Le centre hospitalier St-Joseph de Trois-Rivières, un siècle de dévouement (Trois-Rivières, Comité des fêtes du centenaire du Centre hospitalier St-Joseph de T.-R., 1987), 99 p.; H. E. MacDermot, A History of The Montreal General Hospital (Montréal, Montreal General Hospital, 1950), 135 p.; C. Perreault, Les 100 ans de l'Hôtel-Dieu d'Arthabaska 1884-1984 (Arthabaska, Éditions Pourquoi Pas, 1983), 471 p.; Lewis D. Sclater, Royal Victoria Hospital, 1887-1947 (Montréal, McGill University Press, 1969), 327 p.; Sœur Marie-Madeleine, Centenaire. Hôpital Saint-Joseph, 18641964 (Trois-Rivières, s.é., 1965), 103 p.; Neville Terry, The Royal Vic. The Story of Montreal's Royal Victoria Hospital 1894-1994 (Montréal et Kingston, McGill-Queen's University Press, 1994), 277 p. Certaines études adoptent toutefois des points de vue plus critiques ou nuancés: Denis Goulet, François Hudon et Othmar Keel, Histoire de l'Hôpital Notre-Dame de Montréal. 1880-1980 (Montréal, vlb éditeur, 1993), 432 p.; R. R. Kenneally, The Montreal Maternity, 1843-1926: Evolution of a Hospital, mémoire de maîtrise (histoire), Université McGill, 1983, 163 p.; Normand Perron, Un siècle de vie hospitalière au Québec. Les Augustines et l'HôtelDieu de Chicoutimi 1884-1984 (Québec, Presses de l'Université du Québec, 1984); François Rousseau, La croix et le scalpel. Histoire des Augustines et de l'Hôtel-Dieu de Québec, tome 1: 1639-1892 (Québec, Le Septentrion, 1989), 454 p.

5. En ce qui concerne le rôle de l'État, par exemple, J.-Y. Rivard considère que: «à part les premiers balbutiements de l'hygiène publique au $\mathrm{XIX}^{\mathrm{e}}$ siècle, ce n'est vraiment qu'à partir de l'adoption de la loi de l'assistance publique, en 1921, que l'État commence à jouer un certain rôle dans la distribution des services de santé dans la province. Il faudra cependant attendre encore 40 ans, soit au moment de l'instauration de l'assurance-hospitalisation, avant que l'État devienne le régulateur principal de la distribution des soins.» J.-Y. Rivard et al., «L'évolution des services de santé et des modes de distribution de soins au Québec», Annexe 2, Commission d'enquête sur la santé et le bien-être social (Québec, Éditeur officiel du Québec, 1970), 101.

6. Frédéric Lesemann, Du pain et des services. La réforme de la santé et des services sociaux au Québec (Laval, Éditions coopératives Albert Saint-Martin, 1981), 232 p.; Michel Pelletier et Yves Vaillancourt, Les politiques sociales et les travailleurs, Cahier I: Les années 1900 à 1929 (Montréal, s.é., 1974), 132 p.; Marc Renaud, op. cit.; Yves Vaillancourt, Les politiques sociales et les travailleurs, Cahier II: Les années 1930 (Montréal, l'auteur, 1975), 424 p. 
d'un système demeuré à bien des égards relativement stationnaire depuis la fin du $\mathrm{XIX}^{\mathrm{e}}$ siècle. Qu'elle soit le reflet d'une situation idéale selon les uns, ou néfaste selon les autres, pareille image s'intégrait bien à leurs discours respectifs: alors que les partisans d'une mainmise du clergé et d'organismes privés sur les services sociosanitaires voyaient là un respect salutaire de traditions essentielles à un développement harmonieux de la société québécoise, les promoteurs d'un système hospitalier laïque pris en charge par l'État s'insurgeaient contre ce qu'ils interprétaient comme un retard du Québec en regard des autres sociétés occidentales. C'est précisément cette perception assez largement partagée - bien que l'on puisse parfois trouver des interprétations plus nuancées - d'une institution qui aurait peu évolué que nous entendons réévaluer, et ainsi inscrire notre démarche dans le prolongement des quelques travaux qui, déjà, ont mis en lumière certaines des transformations du système hospitalier québécois durant la première moitié du $\mathrm{XX}^{\mathrm{e}}$ siècle ${ }^{7}$.

Les perspectives d'analyse dominantes en histoire de la médecine avant la décennie 1970 aident à expliquer qu'on ait privilégié au Québec un modèle aussi réducteur. Ici comme ailleurs, les auteurs des monographies d'hôpitaux négligeaient d'inscrire l'établissement dans son cadre socio-économique, si bien qu'il apparaissait comme une entité isolée dont l'évolution était essentiellement fonction de facteurs internes. Divers auteurs, aux États-Unis notamment, ont depuis montré que les transformations de l'hôpital durant les dernières décennies du $\mathrm{XIX}^{\mathrm{e}}$ siècle et les premières du siècle suivant sont tout autant fonction de changements survenus dans la société, et que la redéfinition de l'hôpital en tant que haut-lieu de l'exercice d'une médecine de pointe résulte en bonne partie d'une interaction complexe entre plusieurs facteurs d'ordres économiques, sociaux, démographiques ou politiques $^{8}$. Cette tendance, en histoire de la médecine, à l'intégration de

7. Normand Perron, notamment, a démontré que les médecins au Saguenay cherchaient à accroître leur pouvoir au sein de l'hôpital, ce à quoi les religieuses s'opposaient. Voir op. cit. et «Santé et médecine hospitalière au Saguenay, 1900-1930», Société canadienne d'histoire de l'Église catholique (1988): 75-86.

8. Charles E. Rosenberg, par exemple, considère l'hôpital comme un microcosme reproduisant les valeurs et les rapports sociaux de la société. Voir à ce sujet C. E. Rosenberg, The Care of Strangers. The Rise of America's Hospital System (New York, Basic Books, 1987). Voir aussi David Rosner, A Once Charitable Enterprise. Hospitals and Health Care in Brooklyn and New York, 1885-1915 (Cambridge University Press, 1982); Morris J. Vogel, The Invention of the Modern Hospital (Chicago, University of Chicago Press, 1980); Rosemary Stevens, In Sickness and in Wealth. American Hospitals in the Twentieth Century (New York, Basic Books, 1989); Paul Starr, The Social Transformation of American Medicine. The Rise of a Sovereign Profession and the Making of a Vast Industry (New York, Basic Books, 1982), 514 p. Pour l'Angleterre, on peut également consulter un ouvrage comme celui de John V. Pickstone, Medicine and Industrial Society. A History of Hospital Development in Manchester and its Region, 1752-1946 (Manchester, Manchester University Press, 1985), 369 p. 
diverses perspectives d'analyse est demeurée marginale au Québec en ce qui concerne les travaux portant sur les hôpitaux. Une étude sur l'Hôpital Notre-Dame de Montréal, publiée récemment, souscrit toutefois d'emblée à de telles orientations de recherche. Les auteurs ont pu constater que, dans le cas de cet établissement, en plus de la progression des connaissances médicales et des besoins d'aide humanitaire, d'autres impératifs avaient joué sur l'évolution des services, par exemple une pression démographique croissante en milieu montréalais et la transmission des savoirs médicaux'. L'Hôpital Notre-Dame constitue cependant une exception dans le système hospitalier québécois, dans la mesure où, dès sa création et selon les mots des auteurs, il «apparait comme une combinaison originale de (...) deux modèles hospitaliers ${ }^{10}$ » qui ont influencé le développement des hôpitaux au Québec, combinaison qui le distingue à la fois des autres hôpitaux francophones et des hôpitaux anglophones. Enfin, un autre chercheur, Normand Perron, montre dans ses travaux sur l'Hôtel-Dieu de Chicoutimi que, dans un hôpital situé en région, diverses transformations ont pris place qui s'accordent mal avec l'idée d'un système hospitalier immobile ${ }^{11}$.

Le courant de recherche actuellement dominant en histoire de la médecine et de la santé invite en somme à un élargissement du cadre d'analyse inspiré de l'histoire sociale. Or, pareil élargissement a pour effet d'introduire parmi les intervenants qui ont exercé une influence sur l'évolution de l'hôpital d'autres acteurs que les philanthropes locaux, communautés religieuses et médecins mis en scène par les monographies traditionnelles, notamment la clientèle. Certains de ces intervenants, tels la grande entreprise, les compagnies d'assurance, l'État, ont à notre avis joué un rôle majeur dans cette évolution au Québec durant la première moitié du $\mathrm{XX}^{\mathrm{c}}$ siècle. C'est une assertion que nous avons voulu vérifier en recourant aussi bien aux archives municipales et gouvernementales qu'à celles des établissements hospitaliers.

\section{DIVERSIFICATION DES SERVICES ET PERTE DU MONOPOLE RÉGIONAL DU CLERGÉ AVANT 1920}

Lorsqu'en 1889, les Sœurs de la Providence ouvrirent à TroisRivières un petit hôpital ${ }^{12}$, son organisation était conforme au modèle

\footnotetext{
9. Op. cit.

10. Ibid., 435.

11. Op. cit.

12. L'hôpital trifluvien des Ursulines, qui avait ouvert ses portes en 1702, était fermé depuis 1886 .
} 
traditionnel: tenu par des religieuses et financé surtout par la charité privée $^{13}$, il avait pour principale fonction les soins aux démunis. Rapidement cependant, dans la foulée des transformations qui affectaient le monde hospitalier nord-américain, il allait s'ajuster aux nouvelles exigences de la médecine moderne, non sans que cela crée quelques frictions entre les religieuses et les médecins desservants, toujours désireux d'obtenir l'achat d'équipements récents et l'ouverture de nouveaux services. Nous entendons montrer ici qu'en Mauricie, durant la période allant de la fin du $\mathrm{XIX}^{\mathrm{e}}$ siècle à la fin de la Première Guerre mondiale, les besoins de la grande entreprise en matière de soins médico-hospitaliers allaient jouer un rôle de premier plan dans cette redéfinition de l'hôpital.

Les médecins de l'Hôpital Saint-Joseph furent d'abord étroitement encadrés par le clergé local. Ainsi, ce fut sous le patronage de l'évêque des Trois-Rivières, et fréquemment en présence du curé de la principale paroisse de la ville de même que de la supérieure locale de la communauté, qu'ils tinrent les réunions du Bureau médical jusqu'à la Première Guerre mondiale environ. Diverses clauses des règlements de l'hôpital imposaient aux médecins des contraintes dans l'exercice de leur profession: par exemple, interdiction de révéler aux patients les médicaments utilisés «afin de ne pas faire critiquer leurs prescriptions ${ }^{14}$ », observation d'une "sage économie dans leurs ordonnances» - dont les religieuses devaient assurer la fourniture aux indigents , défense de visiter les patientes sans être accompagné d'une sœur ${ }^{15}$.

Dès la fin du $\mathrm{XIX}^{\mathrm{e}}$ siècle, les médecins allaient cependant revendiquer, parfois obtenir, des changements au sein de l'hôpital, en particulier l'ouverture de nouveaux services et la modernisation des équipements. Une remarque de la religieuse responsable des chroniques de la communauté en 1898 témoigne des pressions qu'ils exerçaient parfois: «Ce rapport est une série de plaintes dans lesquelles il y a du vrai, de l'exagéré et du faux. Nos médecins voudraient qu'on leur fournisse un établissement modèle avec toutes les améliorations du jour ${ }^{16} .{ }^{\prime} C^{\prime}$ 'est à la suite de représentations des médecins auprès des autorités de l'établissement qu'une école de garde-malades fut ouverte en 1907, qu'un premier service ambulancier permanent à Trois-

13. Le gouvernement du Québec donnait également une allocation annuelle qui, à partir de 1909, se chiffra à $2500 \$$.

14. On peut se demander dans quelle mesure les médecins étaient de toutes façons favorables à cette clause limitant l'information donnée au patient.

15. Les obligations des médecins membres du Bureau médical étaient précisées dans les règlements de l'hôpital. Voir Constitutions et règlements de l'hôpital Saint-Joseph des TroisRivières (Trois-Rivières, Presses à vapeur du Trifluvien, 1891).

16. Chroniques des Sœurs de la Providence, 1897-1898. 
Rivières fut mis sur pied, qu'une salle de bactériologie fut aménagée en 1909 et que des dispensaires pour les pauvres et les tuberculeux ouvrirent leurs portes en 1911.

L'exercice d'un pouvoir par les médecins à l'intérieur de l'établissement était certes lié au prestige grandissant de la science médicale. Ainsi, les succès thérapeutiques du médecin-chirurgien en titre, L.-P. Normand, faisaient l'objet de commentaires enthousiastes de la part des religieuses et de ses confrères. Les médecins pouvaient tabler sur leur réputation d'hommes de science pour obtenir de puissants appuis dans la société locale, qui accentuaient les pressions exercées sur les religieuses. En ce qui concerne la création des dispensaires par exemple, un ancien maire, le maire en titre (lui-même médecin), un futur maire de la ville et un patron d'une grande entreprise de textile apportèrent leur concours ${ }^{17}$. Un autre facteur aide à expliquer l'influence grandissante des médecins sur l'évolution des services dispensés: ils étaient devenus les fournisseurs d'une clientèle susceptible d'arrondir les revenus des hôpitaux, c'est-à-dire des patients payant les coûts des chambres privées. Enfin, ils bénéficiaient d'une position morale inattaquable puisque, en retour de l'usage qu'ils faisaient des équipements et des installations, ils soignaient gratuitement les patients des salles communes, dont l'admission était laissée à leur discrétion.

Durant les années 1910, l'influence des médecins allait être renforcée par la perte du monopole du clergé sur les services hospitaliers régionaux. Il y avait bien un hôpital indépendant du clergé à Trois-Rivières depuis 1896. Mais il s'agissait tout autant sinon plus d'un hôtel, d'une station de "villégiature médicale ${ }^{18}$ » où une clientèle fortunée souvent de l'extérieur de Trois-Rivières pouvait dans un cadre agréable se soumettre aux thérapies à la mode. En fait, le sanatorium du docteur C.-N. DeBlois n'était pas concurrent des établissements du clergé, et sa présence n'entraîna pas de débat. Il en alla tout autrement lorsqu'un médecin en froid avec la direction de l'Hôpital Saint-Joseph où il siégeait au Bureau médical, le docteur Georges Bourgeois $^{19}$, ouvrit en 1912 un petit hôpital général. L'évêque, «opposé à cet hôpital laïque ${ }^{20}$ » selon la chronique des Sœurs de la

17. Voir notamment Archives du Séminaire de Trois-Rivières, procès-verbaux des délibérations de l'Association médicale du district des Trois-Rivières des 5 et 22 mai 1911.

18. Voir le prospectus publicitaire Sanatorium Trois-Rivières. 1896-1916.

19. Le docteur Georges Bourgeois appartenait à l'élite médicale québécoise. Membre du Conseil d'hygiène de la province, il avait ainsi participé à la rédaction d'un rapport d'enquête sur la tuberculose en 1910.

20. Chroniques des Sœurs de la Providence, 1911-1912, 129. 
Providence, tenta en vain de bloquer le projet en refusant que des religieuses y agissent comme infirmières. Les rapports du docteur Bourgeois avec l'évêque et la direction de l'Hôpital Saint-Joseph se détériorèrent encore plus lorsqu'il s'adjoignit un associé de religion protestante: C. E. Cross, médecin antérieurement au service de l'entreprise de production hydroélectrique Shawinigan Water and Power.

En dépit des obstacles érigés par le clergé local, l'établissement prospéra rapidement et fut agrandi en 1918. Toutefois, n'eût été de la clientèle ouvrière apportée par les grandes entreprises implantées en Mauricie, l'on peut se demander s'il aurait prospéré autant. En effet, ce fut le docteur Bourgeois qui obtint les contrats d'assurancehospitalisation collective des travailleurs d'usines locales, contrats convoités également par la direction de l'Hôpital Saint-Joseph qui entreprit diverses démarches auprès des entreprises et de leurs employés ${ }^{21}$. Depuis l'adoption de la Loi des Accidents du travail en 1909, les compagnies trouvaient avantage à conclure de telles ententes avec les hôpitaux. En effet, désormais reconnues responsables des accidents de travail survenus dans leurs entreprises, elles cherchaient à obtenir pour leurs employés des services d'urgence efficaces à des tarifs avantageux. Or, l'Hôpital Bourgeois disposait à cet égard d'un atout majeur sur son concurrent: il possédait la seule ambulance automobile de la ville, ce qui lui permettait de véhiculer plus rapidement les accidentés. Dans bien des cas, ces derniers pouvaient ainsi être soignés plus efficacement de sorte que les frais assumés par les compagnies étaient vraisemblablement moins élevés. À l'évêque qui s'inquiétait de la faible fréquentation de l'Hôpital Saint-Joseph à la fin de 1912, le docteur L.-P. Normand répondit notamment: «le Dr Bourgeois exerce un contrôle sur les sociétés, les manufactures, $\& \&^{22}$.» Dans les circonstances, le clergé local dut s'avouer impuissant à attirer cette clientèle dans son propre établissement, comme le montre cette déclaration de l'évêque:

Nous déplorons (...) le sort de nos ouvriers qui sont forcément dirigés à un hôpital où on ne voit point le crucifix, et où les secours de la religion sont négligés. (...) Notons (...) que les sociétaires sont passifs et ne peuvent améliorer leur sort sans s'exposer à compromettre leurs intérêts financiers: toutes démarches en ce sens paraîtraient hostiles aux yeux des chefs des compagnies $^{23}$.

21. Délibérations du bureau médical de l'Hôpital Saint-Joseph, 30 mars 1916, 60; lettre du 10 février 1919 de G. Bourgeois aux maire et échevins, CPTR, février 1919.

22. Ibid., 23 décembre 1913.

23. Ibid., 12 décembre 1916, $20^{\mathrm{e}}$ séance, 64. 
Le cas de Shawinigan atteste avec encore plus d'éloquence de l'intérêt nouveau de la grande entreprise pour le domaine des soins hospitaliers, et de l'incapacité du haut-clergé à contrer ses interventions. Dès 1909, la compagnie fondatrice de cette ville nouvelle de ressources, Shawinigan Water and Power, ouvrit un hôpital entièrement laïque destiné principalement aux cadres et aux travailleurs industriels de la ville. À partir de 1918, d'autres grandes entreprises shawiniganaises collaborèrent à la gestion de l'hôpital. Trois ans plus tard, la compagnie Laurentide Corporation ouvrit à son tour un hôpital à Grand-Mère, ville voisine de Shawinigan. L'évêque des TroisRivières estimait pourtant en 1913 qu'à l'exception de quelques salles pour des cas d'urgence dans des lieux éloignés - il pensait probablement ici au petit hôpital ouvert l'année précédente par des religieuses à La Tuque _ «un seul hôpital [était] suffisant pour le district des Trois-Rivières ${ }^{24}{ }^{-}$. En fait, il s'inquiétait d'une perte de contrôle éventuelle du clergé sur les services hospitaliers, préoccupation clairement évoquée dans les Chroniques des Sœurs de la Providence en 1911-1912: "Mgr craint que les hôpitaux tombent entre les mains des séculiers ${ }^{25}$.» Mais d'autres intervenants que le clergé, malgré ses réticences, avaient déjà pris pied en Mauricie dans le secteur hospitalier.

$\mathrm{Au}$ sein même du grand hôpital régional de Trois-Rivières, l'apparition d'hôpitaux concurrents en Mauricie minait le pouvoir de la communauté religieuse qui en était propriétaire. En effet, les demandes des médecins desservants, dont on craignait vraisemblablement qu'ils fussent tentés d'œuvrer dans ces nouveaux lieux d'exercice de la médecine, acquirent plus de poids. Sous cette pression nouvelle, la direction de l'Hôpital Saint-Joseph chercha durant les années suivantes à harmoniser ses rapports avec les médecins, notamment en se rendant plus aisément à leurs requêtes. En quelques années, on installa des cabinets d'électrothérapie et de radiologie, de même qu'un laboratoire. De plus, on accéda à une demande déjà maintes fois formulée par les médecins et les dames de la ville en ouvrant enfin une maternité: les religieuses craignaient qu'un médecin ne crée l'un de ces petits hôpitaux privés qui, d'après la Chronique des sœurs en 1912-1913, «font dommage aux nôtres en divisant les forces ${ }^{26} \gg$.

24. Ibid., 23 décembre 1913.

25. Chroniques des Sœurs de la Providence, 1911-1912, 140.

26. Ibid., 1912-1913, 166. La majeure partie des informations concernant l'ouverture de nouveaux services provient également des Chroniques. 
Il faut se garder de réduire cette nouvelle dynamique des rapports de pouvoir dans le domaine hospitalier à une simple épreuve de force entre les médecins, tenants d'un hôpital évoluant de concert avec les avancées de la science médicale, et un clergé réfractaire à la modernité. Bien au contraire, les chroniques des religieuses de l'Hôpital Saint-Joseph laissent parfois voir leur satisfaction lors de l'introduction de nouveaux services ou équipements. Elles avaient par ailleurs des alliés influents parmi les médecins, en particulier le docteur L.-P. Normand, membre de l'élite médicale de la province élu président du Collège des médecins et chirurgiens de la province de Québec en 1907, lui-même tenant d'une modernisation des services médicohospitaliers ${ }^{27}$. Ce fut d'ailleurs celui-ci qui, sur une suggestion de l'évêque trifluvien semble-t-il ${ }^{28}$, se porta en 1919 acquéreur de la part du docteur Bourgeois (gravement malade) dans le petit hôpital laïque, ce que les religieuses saluèrent avec enthousiasme.

L'accueil souvent favorable des religieuses aux nouveautés médicales, leurs alliances avec des personnages influents amélioraient sans doute leurs relations avec les médecins de l'Hôpital Saint-Joseph, mais les pressions qu'elles subissaient en raison de l'existence d'autres établissements de soins ne cessèrent pas pour autant. Ainsi, la concurrence de l'ancien Hôpital Bourgeois, rebaptisé Normand \& Cross, demeura très vive. Il est vrai qu'un incident vint assombrir les rapports de monsieur Normand avec l'Hôpital Saint-Joseph. En effet, en 1921, lorsqu'il fut nommé ministre au gouvernement fédéral, les religieuses refusèrent la demande qu'il leur fit d'être remplacé lors de ses absences comme chirurgien par son associé de l'hôpital concurrent, le docteur protestant C. E. Cross ${ }^{29}$. Les religieuses, auxquelles on reprocha de ne pas avoir appuyé leur bienfaiteur (dons de matériel notamment) lors des élections fédérales suivantes, firent ce constat: «le mouvement des malades, tant chez les femmes que chez les hommes s'est quelque peu ralenti. Que de cas payants vont à l'hôpital Normand! $!^{30}$ ».

L'apparition et la concurrence d'hôpitaux - même petits ${ }^{31}$ indépendants du clergé ne représentaient pas seulement une perte de

27. Le docteur L.-P. Normand était chirurgien de l'hôpital des religieuses et chef de file des conservateurs de la ville. Il fut élu maire à quelques reprises.

28. C'est du moins ce qu'affirme la chronique des Sœurs de la Providence: «Monseigneur l'Évêque qui a constamment l'œil ouvert sur les intérêts spirituels de ses ouailles et craignant que cet hôpital ne passe à des autorités protestantes et pis encore, suggère au $\mathrm{D}^{r}$ Normand d'acheter l'immeuble. Notre bon chirurgien se rend aux désirs de son Évêque.» Chroniques des Sœurs de la Providence, 1919-1920, 450.

29. Ibid., 1921-1922, 4: 48-49, 58 .

30. Ibid., 1922-1923, 4: 99.

31. L'Hôpital Bourgeois ne comptait que 13 lits lors de son ouverture, capacité doublée en 1918, et celui de Shawinigan entre dix et vingt lits avant cette année, lorsqu'il fut aussi agrandi. 
contrôle de celui-ci sur les services hospitaliers, mais également une perte financière. En effet, ces hôpitaux canalisaient une partie de la clientèle payante, laquelle constituait une source de revenus de première importance pour les hôpitaux ${ }^{32}$. Les ouvriers «sociétaires» (couverts par une assurance collective), mais aussi des patients en mesure de payer isolément leurs frais de séjour à l'hôpital, pouvaient désormais se tourner vers ces établissements laïques. Or, on sait que pour s'ajuster à une médecine hospitalière en pleine transformation, les hôpitaux devaient consentir des dépenses bien plus considérables qu'auparavant en achats d'équipements, en réaménagements internes, en personnel qualifié, etc. Dans un contexte où les établissements du clergé comme l'Hôpital Saint-Joseph tiraient encore l'essentiel de leurs revenus de la charité privée, il n'y a rien d'étonnant à ce que leur direction et le haut-clergé se soient montrés défavorables à la création d'hôpitaux concurrents accueillant une partie de la clientèle payante. À partir des années 1920 cependant, l'État allait augmenter substantiellement son aide aux établissements dispensant des soins aux démunis.

\section{FINANCEMENT PAR L'ÉTAT DES SOINS AUX INDIGENTS ET DE L'EXTENSION DES INFRASTRUCTURES HOSPITALIÈRES DURANT L'ENTRE-DEUX GUERRES}

Après la Première Guerre mondiale, on admettait généralement que les grands hôpitaux québécois n'avaient plus les ressources financières pour respecter les nouvelles normes nord-américaines en matière d'organisation des services, notamment en ce qui a trait au renouvellement des équipements. Ceci était d'autant plus patent que les hôpitaux devaient répondre à une demande croissante de services en milieu urbain, tout en continuant de prendre soin gratuitement des plus démunis. Une voie paraissait viable: obtenir des pouvoirs publics qu'ils prennent à leur charge les frais reliés à cette dernière fonction. C'est ainsi qu'en 1919, six hôpitaux montréalais envoyèrent aux municipalités de la province une circulaire expliquant que seuls les malades indigents dont la municipalité d'origine aurait accepté à l'avance de payer les dépenses d'hôpital seraient $\operatorname{admis}^{33}$. Puis en 1921, le gouvernement libéral de L.-A. Taschereau adopta une loi qui allait assurer pendant environ quatre décennies le financement de

32. Voir à ce sujet D. Goulet, F. Hudon et O. Keel, op. cit., 69.

33. Commissions permanentes, archives municipales de Trois-Rivières, 15 septembre 1919, circulaire du $1^{\text {er }}$ mai 1919 Aux municipalités de la province de Québec, signée par les autorités de l'Hôpital Notre-Dame, du Montreal General Hospital, du Royal Victoria Hospital, de l'Hôpital Sainte-Justine, du Western Hospital et de l'Hôtel-Dieu. 
l'hébergement des indigents dans divers types d'établissements hospitaliers.

La Loi de l'Assistance publique a déjà été abondamment commentée ${ }^{34}$. Déjà, dans les années suivant son adoption, elle donna lieu à un vibrant débat où la presse catholique, Henri Bourassa en tête, combattit l'ingérence de l'État dans le domaine hospitalier. Ces défenseurs du système traditionnel d'aide sociale réclamaient que l'État finance les soins aux indigents sans surveiller ou réglementer l'usage fait des subventions, ce à quoi s'opposa le gouvernement. Après la Seconde Guerre mondiale, cette loi a également été critiquée par divers intellectuels, puis, depuis les années 1960 et 1970 surtout, par des historiens. Mais alors que les opposants de la première heure avaient insisté sur les pouvoirs à leur avis exhorbitants que l'État s'octroyait, les intellectuels se sont depuis le plus souvent appliqués à souligner la timidité de son intervention. Tant et si bien que la Loi de l'Assistance publique est souvent considérée aujourd'hui comme le moyen trouvé par une petite bourgeoisie conservatrice de maintenir intact un système hospitalier traditionnel évoluant sous la houlette du clergé $^{35}$ et ce, bien que certains tenants d'un tel système l'aient vigou-

34. Voir Henri Bourassa, Une mauvaise loi. L'assistance publique (Montréal, Imprimerie du Devoir, 1921); Deuxième rapport de la Commission des assurances sociales de Québec, janvier 1932; Arthur Saint-Pierre, L'œuvre des congrégations religieuses de charité: dans la province de Québec (Montréal, Éd. de la Bibliothèque canadienne, 1932); Esdras Minville, Le régime social dans la province de Québec, étude préparée pour la Commission royale des relations entre le Dominion et les provinces (1939); H. M. Cassidy, Public Health and Welfare Reorganization. The Postwar Problem in the Canadian Provinces (Toronto, The Ryerson Press, 1945); G. Poulin, L'assistance sociale dans la province de Québec, 1608-1951 (Commission royale d'enquête sur les problèmes constitutionnels, 1955); Rapport du comité d'étude sur l'assistance publique (Gouvernement du Québec, 1963); Serge Mongeau, Évolution de l'Assistance au Québec (Montréal, Éditions du Jour, 1967), 123 p.; J.-Y. Rivard, op. cit.; Antonin Dupont, Les relations entre l'Église et l'État sous Louis-Alexandre Taschereau, 1920-1936 (Montréal, Guérin, 1972); Michel Pelletier et Yves Vaillancourt, Les politiques sociales et les travailleurs, cahier I: Les années 1900 à 1929 (Montréal, s.é., 1974); Hervé Anctil et Marc-A. Bluteau, «La santé et l'assistance publique au Québec 1886-1986», Santé Société, numéro spécial (1986).

35. D'après Frédéric Lesemann, cette loi «contribue essentiellement à renforcer les pouvoirs établis clérico-petits-bourgeois et l'ordre social existant», op. cit., 22. Serge Mongeau estime quant à lui que la Loi de l'Assistance publique était «fort improprement nommée car cette loi ne visait aucunement à fournir une assistance directe aux indigents, mais à assister les institutions privées pour leur donner les moyens de continuer à aider gratuitement les indigents», op. cit., 45. Marc Renaud écrit que cette loi «was an ingenious means of supporting religious communities and charitable institutions while not impinging upon the very concept of charity...», op. cit., 46. Il reprenait ainsi l'évaluation faite par J.-Y. Rivard, op. cit., 21. Quant à Esdras Minville, il considérait en 1939 que «l'État dut se décider à aider financièrement mais de telle manière que son intervention ne bouleversât pas le régime établi et à l'édification duquel il n'avait pas contribué», Esdras Minville, Syndicalisme, législation ouvrière et régime social au Québec avant 1940 (Montréal, Presses HEC et Fides, 1986), 291. Des auteurs comme Michel 
reusement combattue durant la décennie 1920. Il est certes exact que l'autorité du clergé à l'intérieur de ses établissements n'était que fort peu entamée directement par la loi $^{36}$. Mais l'on verra que le clergé se voyait imposer diverses contraintes pour user de l'aide étatique, et que le Service de l'Assistance publique (SAP), organisme gouvernemental créé en vertu de la loi, était en mesure d'exercer sur lui des pressions, ne serait-ce que par l'attribution discrétionnaire de subventions aux fins de construction ou d'agrandissement ${ }^{37}$.

Les revenus alimentant le fonds de l'Assistance publique provenaient de taxes sur les divertissements, puis sur les repas au restaurant dès 1926 et des versements de la Commission des liqueurs de la province à partir de 1929. Ils se chiffraient en millions de dollars à répartir dans le réseau hospitalier de la province, soit pour financer les soins donnés aux indigents dans diverses sortes d'établissements, soit à titre de subventions destinées principalement à l'extension et au renouvellement des infrastructures hospitalières. Dans le premier cas, toute une série de modalités étaient définies auxquelles allaient se plier les établissements pour obtenir l'argent du fonds de l'Assistance publique. Ils devaient d'abord, après en avoir fait la demande, être admis comme institution d'Assistance publique. Cette autorisation

Pelletier et Yves Vaillancourt ont cependant reconnu que cette loi représentait une petite brèche dans un secteur antérieurement contrôlé par la petite-bourgeoisie, brèche susceptible d'être élargie par la suite. Voir op. cit., 100 et suivantes. Enfin, H. Anctil et M.-A. Bluteau, tout en indiquant que l'État s'assurait par le financement "une certaine forme de contrôle», insistent sur le fait que la loi respectait les traditions, en réaffirmant «le statut privé de l'assistance, tout en reconnaissant à l'indigence un caractère public», op. cit., 56.

36. Un rapport d'enquête gouvernementale décrit ainsi en 1963 l'organisation du bienêtre dans la province: «Comme les problèmes sociaux, à cause de leur amplitude, conduisaient à un transfert inévitable de responsabilités, l'État, dont les pouvoirs effectifs s'accroissaient, n'a pas voulu modifier organiquement le secteur privé. Au lieu de participer lui-même directement à l'administration des sommes versées en assistance sociale, il a préféré laisser cette administration aux agences privées. Ainsi demeurait sauve l'image traditionnelle de l'organisation du bien-être dans la société québécoise. La notion du rôle supplétif de l'État se trouvait officiellement protégée, puisque les attributions du secteur privé demeuraient considérables, malgré la présence de problèmes sociaux qu'il aurait été incapable de résoudre par ses propres moyens financiers. (...) Cette solution particulière a doté le Québec d'une organisation administrative composite qui ne relève entièrement ni du secteur public ni du secteur privé.» Gouvernement du Québec, Rapport du comité d'étude sur l'assistance publique (juin 1963), 108-109.

37. Tout en insistant sur le peu de pouvoir que s'accordait l'État sur les institutions privées, certains auteurs ont par contre reconnu que la Loi de l'Assistance publique introduisait des modifications peut-être plus importantes que sa seule lecture ne le laissait entrevoir. Ainsi, J.-Y. Rivard écrit: «Toutefois l'assistance publique, par ses subventions, n'a pas été sans influencer profondément les modes de distribution des soins dans la province. L'assistance publique aura peut-être été un stade intermédiaire, précurseur d'un système plus global d'organisation sociale devant la maladie.» J.-Y. Rivard et al.., op. cit., 61 . 
était accordée après une enquête au cours de laquelle le Service de l'Assistance publique pouvait exiger toute l'information qu'il jugeait utile. L'admission des indigents devait respecter tout un ensemble de procédures, requérait la tenue d'un registre des indigents admis, l'envoi de formulaires, puis de rapports. Dans le cas de l'hospitalisation, un responsable municipal devait signer l'un de ces formulaires, de même qu'un médecin. L'autorisation de ce dernier était indispensable, puisque les médecins allaient dispenser leurs soins gratuitement aux bénéficiaires de l'aide gouvernementale. Les coûts d'entretien des indigents étaient fixés par le gouvernement selon une grille organisée par type et par taille d'établissements, et subdivisés en trois parts en principe égales, la première étant payable par le gouvernement et la seconde, par la municipalité, l'établissement assumant le reste des dépenses. Les parts du gouvernement et de la municipalité étaient fixes alors que celle de l'établissement variait selon les dépenses réellement effectuées. Enfin, la loi autorisait le SAP à visiter les locaux où étaient hébergés et soignés les indigents.

Si le SAP demeura très conciliant dans l'application de la loi durant les années 1920, il devint plus rigoureux durant la décennie suivante. Cherchant à contenir une hausse rapide de ses dépenses (voir figure 1), il adopta diverses mesures visant à réduire ses versements aux établissements. Certains, comme l'Hôpital Saint-Joseph de TroisRivières, furent rétrogradés de classe de sorte qu'ils bénéficièrent de tarifs moins avantageux ${ }^{38}$. L'Hôpital Sainte-Thérèse de Shawinigan, ouvert en 1931, n'obtint pas d'être classé A-1 comme les religieuses l'avaient souhaitée ${ }^{39}$. Surtout, le Service engagea un inspecteur chargé de parcourir la province pour voir au respect de la $10 i^{40}$, et notamment à ce que les indigents ne soient pas classés dans des catégories à tarifs trop élevés. Les tarifs pour un lit d'hospice, par exemple, étaient nettement moins élevés que pour un lit d'hôpital: ainsi, durant les premières années, si le total des contributions de la municipalité et du gouvernement était de $0,30 \$$ par jour pour un vieillard hébergé dans un hospice, elles atteignaient respectivement $1,34 \$, 1,00 \$$ et $0,70 \$$ pour des malades reçus dans des hôpitaux généraux de classe $\mathrm{A}$ (sanatoriums antituberculeux y compris), B ou C. La nouvelle rigueur avec laquelle on entendait surveiller la classification des malades contribua

38. Nous savons ainsi qu'un hôpital à Sherbrooke et un à Hull subirent le même sort.

39. Voir à ce sujet la correspondance entre le SAP et l'Hôpital Sainte-Thérèse conservée aux Archives nationales du Québec à Québec.

40. Rapport annuel du Service de l'Assistance publique, 1934-1935, dans Rapport annuel du secrétaire et registraire, 162 . 


\section{FIGURE 1}

\section{Répartition des montants distribués par le gouvernement provincial pour l'assistance publique de 1922 à 1938, en dollars courants et en \%}
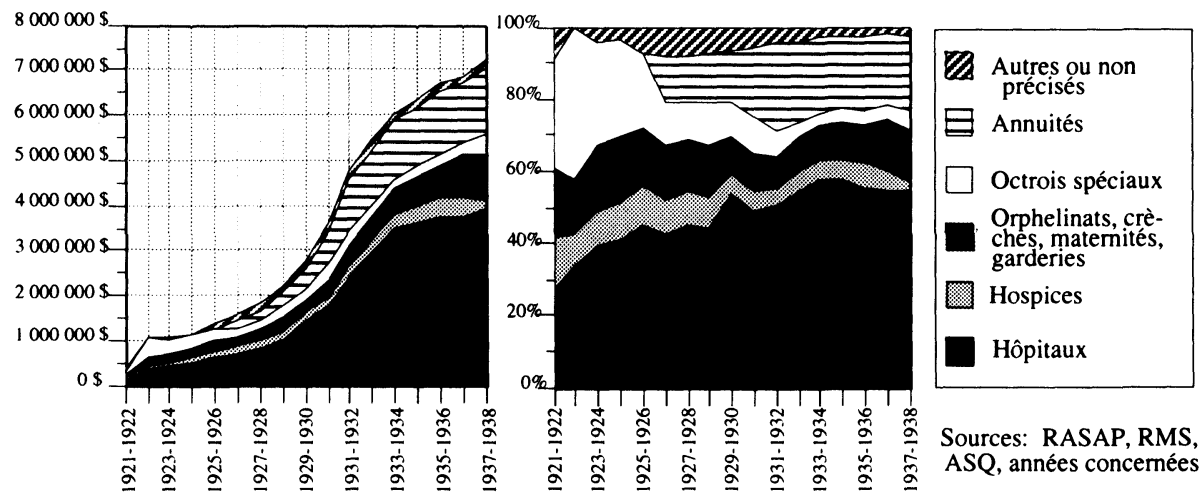

à détériorer les relations entre le SAP et la direction de l'Hôpital Saint-Joseph durant cette période difficile ${ }^{41}$.

Le SAP disposait d'un autre outil pour effectuer des pressions sur les autorités des établissements: un article de la loi prévoyait le versement de subventions d'aide exceptionnelles. Aucun critère n'était fixé pour l'attribution des sommes disponibles, laissant au gouvernement toute latitude et ouvrant la porte au patronage politique, ce qui fut dénoncé en diverses occasions par les contemporains ${ }^{42}$. Et de fait, il fallait non seulement l'accord du SAP, mais également du premier ministre de la province avant que ce type d'aide ne soit accordé. Les subventions aux fins de construction attribuées à Trois-Rivières et à Shawinigan avant 1940 furent toutes autorisées par L.-A. Taschereau ou Maurice Duplessis: plus de 320 000\$ vers 1930 à une société privée laïque pour le sanatorium antituberculeux régional Cooke de Trois-Rivières dont le financement de la construction fut assumé

41. L'extrait de lettre suivant montre bien l'existence de tensions entre le SAP et la direction de l'Hôpital Saint-Joseph: «Je désire que tu ne les manques pas et que tu exiges incessamment quand tu iras, de faire passer cette malade dans une classe inférieure si elles ne réussissent pas à la transférer dans un hospice ou chez des incurables. Combien doit-il y en avoir de ces cas où nous nous faisons exploiter?», Archives nationales du Québec à Québec, Fonds des Affaires sociales, série Hôpitaux du Québec, correspondance de l'Hôpital Saint-Joseph, lettre du 21 février 1935 du directeur du Service de l'Assistance publique à L.-A. Lessard, inspecteur.

42. Voir par exemple H. M. Cassidy, Public Health and Welfare Reorganization. The Postwar Problem in the Canadian Provinces (Toronto, The Ryerson Press, 1945), 382. 
presque en totalité par les pouvoirs publics, plus précisément par le Service de l'Assistance publique et les municipalités de Shawinigan et de Trois-Rivières ${ }^{43} ; 22.5000 \$$ à la municipalité de Shawinigan pour l'érection de l'Hôpital général Sainte-Thérèse qui ouvrit ses portes en $1931^{44}$ et $150000 \$$ en 1939 aux Sœurs de la Providence de l'Hôpital Saint-Joseph pour la construction d'un nouvel hôpital ${ }^{45}$.

Plusieurs exemples montrent que le clergé perdait aux mains du gouvernement provincial le contrôle du développement des infrastructures hospitalières en Mauricie, perte de contrôle dont la première étape avait été, avant 1920, l'apparition d'hôpitaux laïques concurrents. S'il fallut attendre la fin des années trente avant que l'administration de l'Hôpital Saint-Joseph obtienne une subvention pour finalement construire cet hôpital «moderne» que réclamaient les médecins depuis la fin de la Première Guerre mondiale, ce n'était pas faute de tentatives des religieuses ou de l'évêque, mais bien plutôt en raison de désaccords avec le SAP quant à l'usage des sommes devant être attribuées. Celui-ci distribuait l'argent, pour l'essentiel, à ses conditions. Ainsi, pour l'Hôpital Saint-Joseph, une première série de négociations eurent lieu en 1923 qui achoppèrent après que les religieuses aient décidé de modifier en simple agrandissement le projet de construction d'un nouvel hôpital présenté par l'évêque, auquel le SAP avait donné son accord ${ }^{46}$. Quelques années plus tard, un projet de sanatorium pour tuberculeux donna lieu à une épreuve de force entre le SAP et l'évêque. Ce dernier avait d'abord approuvé en 1925 l'érec-

43. Les informations sur l'hôpital Cooke proviennent essentiellement de la correspondance du SAP conservée dans les Archives nationales du Québec à Québec, des procèsverbaux des délibérations de la Corporation de l'Hôpital Cooke conservés à l'hôpital même, et d'articles de journaux.

44. Les données concernant la genèse de l'hôpital Sainte-Thérèse proviennent surtout de la correspondance du SAP conservée aux Archives nationales du Québec à Québec. Voir aussi les procès-verbaux du conseil de ville de Shawinigan, 3 mars 1926; 19 mai 1926; 3 juillet 1929; 21 août 1929; 4 septembre 1929; 5 février 1930; 26 mars 1930; 2 avril 1930; 16 avril 1930; 7 mai 1930; 21 mai 1930; 26 mai 1930; 4 février 1931.

45. Archives nationales du Québec à Québec, correspondance de l'Hôpital Saint-Joseph, lettres des 9 mars, 3 avril, 2 décembre 1937, 16 mai, 9 et 22 juillet 1938 et 11 février 1939 de la supérieure générale de la maison mère des Sœurs de la Providence à J.-H.-A. Paquette; lettre du 28 août 1937 de Émile Nadeau à R. Veilleux; lettres des 3 mars et 25 avril 1938 du sousministre de la santé à la supérieure générale; lettres de la supérieure générale des 21 avril 1938 et 11 février 1939 à Maurice Duplessis; lettre du 7 mars 1939 de l'assistante générale de la communauté à J.-H.-A. Paquette et des 7 mars et 3 avril 1939 au sous-ministre; lettre du 11 mai 1939 de la supérieure de l'hôpital Saint-Joseph au sous-ministre; Chroniques des Sœurs de la Providence, 1938-1939, 341 et suivantes.

46. Archives nationales du Québec à Québec, correspondance de l'Hôpital Saint-Joseph, lettre du 26 juillet 1923 de A. Lessard à monseigneur Massicotte; lettre du 23 juin 1923 de L.A. Taschereau à A. Lessard; lettre du $1^{\text {er }}$ août 1923 de monseigneur Massicotte à A. Lessard; lettre «personnelle» du 3 septembre 1923 de monseigneur Massicotte à A. Lessard. 
tion d'un sanatorium qui aurait été la propriété d'une corporation privée laïque, puis s'était rapidement ravisé pour favoriser plutôt le rajout d'une aile pour tuberculeux à l'Hôpital Saint-Joseph, donc à une institution appartenant à une communauté religieuse (les Sœurs de la Providence firent d'ailleurs en 1925 une autre demande de fonds au $\mathrm{SAP}^{47}$ ). La direction du SAP refusa la demande de l'évêque, alléguant que les établissements de ce genre devaient, selon les données modernes de la médecine, être situés en périphérie des villes; mais l'organisme ne put réaliser son propre projet car, sans l'accord de l'évêque, il était impossible d'obtenir les services d'une communauté religieuse pour gérer l'établissement. Ce fut l'impasse pendant quatre ans, jusqu'à ce que l'évêque baisse pavillon devant les pressions d'hommes publics locaux ou extérieurs à Trois-Rivières, tel le président de l'Association canadienne antituberculeuse ${ }^{48}$.

Une fois les subventions accordées, le SAP se réservait de plus le droit d'examiner pour approbation les plans de construction que devaient lui soumettre les réalisateurs des projets, ce que nous avons pu vérifier dans les cas de la construction de l'Hôpital Sainte-Thérèse en 1930 et du nouvel Hôpital Saint-Joseph en 193949. Pour l'édification de ce dernier, et en vertu de nouvelles règles du SAP, un inspecteur veilla non seulement à ce que les plans soient conformes aux objectifs du Service, mais également, par des visites durant les travaux, à ce que les plans soient bien respectés.

Le gouvernement provincial disposait donc, par l'attribution discrétionnaire de subventions pour la construction, d'un puissant moyen de pression sur les autorités des hôpitaux. Surtout, il s'octroyait un important pouvoir décisionnel sur l'extension et la modernisation des services hospitaliers, quitte à en laisser la gestion à d'autres intervenants. L'envergure des moyens déployés mérite d'être soulignée. Comme le montre la figure 1, le deuxième plus important poste de dépenses du Service de l'Assistance publique, après le financement des soins d'hôpitaux dispensés aux nécessiteux (plus de

47. Archives nationales du Québec à Québec, correspondance de l'Hôpital Saint-Joseph, rapport du 21 décembre 1925 de l'inspecteur de l'Assistance publique Azarie Lessard à A. Lessard, directeur.

48. Une autre tentative des religieuses en vue d'obtenir des fonds pour la construction d'un nouvel hôpital, appuyée par l'évêque et par les principaux leaders politiques trifluviens, se heurta au manque de ressources du Service de l'Assistance publique durant la crise. Voir Archives nationales du Québec à Québec, correspondance de l'Hôpital Saint-Joseph, requête du 18 mars 1931 adressée à Athanase David; lettre de A. Lessard à la supérieure de l'hôpital SaintJoseph.

49. Les plans et devis de l'hôpital Saint-Joseph de Trois-Rivières furent ainsi attentivement examinés par un inspecteur-architecte. 
FIGURE 2

Montants en millions de $\$$ courants des annuités versées
et des emprunts garantis par le Service
de l'Assistance publique, 1925-1939

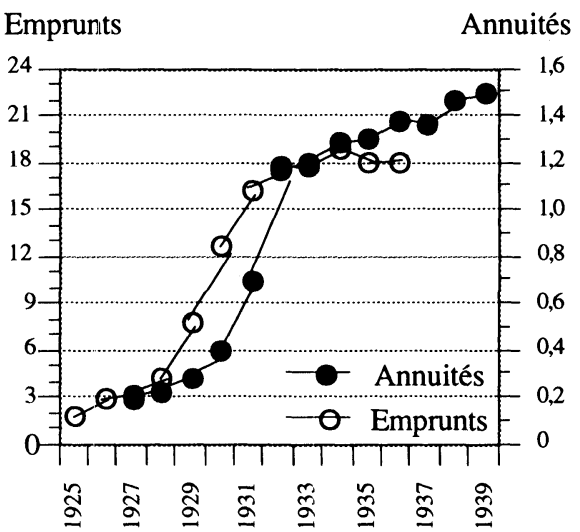

Source: Rapports annuels du SAP, années concernées

3 millions de dollars annuellement à partir de 1933 environ), fut précisément le volet des subventions. Sous forme d'octrois spéciaux dans un premier temps, puis d'annuités garantissant les emprunts contractés par les établissements (intérêts et fonds d'amortissement), plus d'un million de dollars par année à partir de 1931 (figure 2) furent ainsi dépensés pour la construction ou l'agrandissement d'hôpitaux, d'hospices, d'orphelinats, de crèches et de maternités. La principale portion (quoique cette portion reste à être déterminée) était manifestement réservée aux hôpitaux où l'on trouvait d'ailleurs des équipements plus coûteux que dans les autres types d'établissements concernés. Ceci donne une idée du rythme auquel l'on entendait promouvoir le développement du réseau hospitalier. Au tout début des années 1930, par exemple, construire un hôpital général de 100 lits (75 lits pour malades et 25 lits d'hospice) comme l'Hôpital Sainte-Thérèse représenta une dépense de l'ordre de $450000 \$$. Ces chiffres à l'appui, on peut estimer que l'État finançait alors la construction de l'équivalent d'un hôpital de 100 lits par année dans la province, peut-être même plus.

Il nous paraît important d'insister ici sur le programme de subventions aux fins de construction de l'Assistance publique, dans la mesure où les monographies d'hôpitaux parues au Québec n'accordent pas, à notre avis, toute l'importance souhaitable à cet effort financier 
du gouvernement, réduisant ainsi le rôle de l'État dans la constitution du système hospitalier québécois. L'ouvrage de Normand Perron fait toutefois exception en mentionnant les montants accordés à l'HôtelDieu de Chicoutimi, et en soulignant l'importance de ce nouveau mode de financement des constructions d'hôpitaux ${ }^{50}$. Quant à la monographie récente de Denis Goulet et de ses collaborateurs ${ }^{51}$, ses auteurs ont fort justement attiré l'attention sur l'implication croissante de l'État dans le domaine hospitalier par le financement des soins aux indigents, tout en mettant en évidence l'insuffisance des tarifs de l'Assistance publique, sans mentionner par contre les montants de $250000 \$$ en $1925-1926,500000 \$$ en $1929-1930$ et $250000 \$$ en $1930-$ 1931 accordés à l'Hôpital Notre-Dame spécifiquement aux fins de construction.

Le clergé, désormais soumis à la concurrence d'organisations laïques et ayant en partie cédé à l'État la capacité de décider des nouveaux développements des infrastructures hospitalières, conservait toutefois pour l'essentiel son pouvoir décisionnel à l'intérieur de ses établissements et ce, malgré des concessions faites aux médecins. Ainsi, le directeur du SAP ne parvint pas à obtenir de la corporation privée propriétaire du sanatorium Cooke que sa direction soit confiée à un médecin plutôt qu'aux religieuses, comme il l'avait exprimé dans une lettre:

Dans mon opinion, pour que les choses marchent bien, le Service médical doit avoir non seulement le contrôle du traitement des malades, mais doit être l'officier responsable au Comité pour tout ce qui regarde l'administration. Il doit évidemment travailler en coopération avec les religieuses mais, dans mon opinion, c'est lui qui doit être le chef ${ }^{52}$.

Le refus des religieuses de l'Hôpital Saint-Joseph de respecter diverses recommandations visant à réduire la mortalité qui sévissait à la crèche de l'hospice illustre aussi assez bien l'autonomie des autorités des établissements en ce qui a trait à la gestion interne. Le médecin officier de santé de la municipalité de 1923 à 1927 avait souligné à plusieurs reprises le problème de la mortalité élevée à la crèche et, encouragé en cela par le directeur du Service de l'Assistance publique, avait demandé aux médecins desservants qu'ils interviennent auprès des religieuses. La direction de l'établissement répon-

50. Op. cit., 139 et $211-212$.

51. Op. cit.

52. Archives nationales du Québec à Québec, correspondance de l'Hôpital Cooke, lettre du 26 novembre 1929 de A. Lessard à P. Bigué, président de la Corporation de l'Hôpital Cooke. 
dit aux médecins que la crèche faisait partie de l'hospice ${ }^{53}$ et qu'à ce titre elle échappait au contrôle du comité médical ${ }^{54}$. Plus tard, durant la crise des années trente, le problème prit des proportions inquiétantes, le nombre d'enfants admis ayant augmenté rapidement. D'après A. R. Foley, chef de la division de l'épidémiologie du SPH, sur 240 nourrissons admis en 1934 (la pire année), 225 étaient décédés ${ }^{55}$. L'abbé C.-E. Bourgeois qui avait charge du placement des enfants parlait quant à lui de 214 décès sur 320 enfants $(230$ admis durant l'année et 90 déjà présents), soit 2 sur $3^{56}$. Quelques années plus tôt, dans une lettre interne du Service provincial d'hygiène, le directeur du SAP parlait déjà de la crèche en termes de «dépotoir où crèvent les nourrissons qui ont le malheur d'y être recueillis ${ }^{57}$ ».

La direction de l'hôpital estimait que la mortalité exagérée à la crèche était due au piètre état des enfants au moment de leur admission, et l'abbé C.-E. Bourgeois, le responsable du placement des enfants, dénonça la dégradation des mœurs qui était à son avis responsable de tant de naissances illégitimes de même que des ravages de la syphilis congénitale, identifiée comme la principale cause de décès à la crèche ${ }^{58}$. Au Service de l'Assistance publique et au Service provincial d'hygiène, on y voyait plutôt le résultat d'un non-respect de règles d'hygiène élémentaires comme l'usage de lait sain.

Les intervenants extérieurs à la communauté religieuse, incluant le Service de l'Assistance publique qui finançait les soins, ne pouvaient donc exiger quelque modification que ce soit à la gestion de la crèche. Certains allaient cependant gagner en autorité, si bien qu'à la fin de

53. Bien que la crèche et l'hospice soient logés dans le même établissement que l'hôpital et subventionnés par l'État, ils relevaient exclusivement des religieuses. Sur la question de la cohabitation des fonctions caritatives et médico-hospitalières, de même que sur les rapports entre le SAP et la direction de la crèche, voir la thèse de l'auteur, op. cit., 202-203 et 375-380.

54. Délibérations du bureau médical de l'Hôpital Saint-Joseph, 1925, 128; 1926, 135; 1930, 275; 1932, 301; Archives municipales de Trois-Rivières, procès-verbal de la réunion du comité du Centre de démonstration du 27 février 1925.

55. Archives nationales du Québec à Québec, correspondance de l'Hôpital Saint-Joseph, rapport du 7 novembre 1935 de la division de l'épidémiologie concernant l'hôpital.

56. Rapport du directeur de l'organisme Le placement de l'orphelin pour l'année 1934 dans Archives municipales de Trois-Rivières, Procédés du conseil, février 1935.

57. Archives nationales du Québec à Québec, correspondance du Conseil d'hygiène de la province de Québec, boîte correspondance 1924-1950, chemise intitulée «correspondance 19241940", lettre du 9 octobre 1930 du directeur du Service provincial d'hygiène à Paul Parrot, chef de la division de la statistique démographique.

58. Il s'agit en fait de la syphilis congénitale, qui n'est pas héréditaire mais contractée lors de la grossesse par le fotus. Durant les années où le nombre de décès à la crèche devint très élevé, le médecin rattaché à ce service attribua jusqu'à la moitié des décès à cette maladie, ce qui paraît nettement exagéré. Voir les rapports annuels rédigés par C.-E. Bourgeois pour l'CEuvre du Placement de l'orphelin durant les années 1930, et dont des exemplaires peuvent être trouvés dans les Archives municipales de Trois-Rivières. 
la crise les religieuses durent se soumettre à certaines exigences. En effet, après diverses pressions de l'unité sanitaire trifluvienne, établie en 1938, et le remplacement du médecin attitré à la crèche, au moment aussi où se négociait avec succès l'obtention tant attendue de la subvention pour la construction d'un nouvel hôpital, la direction de l'établissement finit par se rendre aux demandes des hygiénistes et du SAP. En quelques années, la mortalité à la crèche diminua considérablement pour atteindre un taux d'environ 5\% en 1941. Le Département de la Santé et du Bien-être social avait manifestement gagné un droit de regard sur les soins dispensés aux nourrissons. Ainsi, en 1939, l'inspecteur du Service de l'Assistance publique exigea de la directrice des explications sur une brusque flambée de décès ${ }^{59}$. De plus, la Supérieure de l'établissement indiqua dans une lettre que les autorités de l'unité sanitaire ne permettaient plus «d'entasser les bébés comme pendant les années 1934 à $1938^{60} \gg$. Enfin, les rapports annuels de l'unité sanitaire adressés à la municipalité de Trois-Rivières montrent que son directeur intervenait désormais auprès de la direction de l'Hôpital Saint-Joseph en ce qui a trait à la crèche.

En somme, peu à peu, la direction de l'Hôpital Saint-Joseph dut admettre que l'Etat exerce une surveillance sur l'usage des subventions, et par conséquent sur l'organisation des services sociosanitaires en milieu hospitalier. Cette surveillance était la contrepartie des avantages considérables de la Loi de l'Assistance publique pour les grands hôpitaux. La loi, en effet, assurait aux communautés propriétaires une certaine stabilité financière et leur permettait d'agrandir et de moderniser leurs établissements. Mais cet outil de transformation et de développement était également pour l'État un outil de contrôle. En Mauricie, la direction du SAP en usa comme d'un levier dans ses négociations avec le clergé et se fit même parfois l'avocat d'une laïcisation de la direction et de la propriété des hôpitaux.

\section{LA FRÉQUENTATION DES HÔPITAUX ET LA DÉPENDANCE DES COMMUNAUTÉS PROPRIÉTAIRES ENVERS L'ÉTAT ET LES MÉDECINS}

Un autre moyen d'apprécier dans quelle mesure le clergé devenait dépendant de l'État et des médecins dans le domaine hospitalier consiste à examiner la fréquentation des hôpitaux trifluviens et shawi-

59. Archives nationales du Québec à Québec, correspondance de l'Hôpital Saint-Joseph, lettre du 22 avril 1940 de la supérieure au sous-ministre de la santé Jean Grégoire.

60. Archives nationales du Québec à Québec, correspondance de l'Hôpital Saint-Joseph, octobre 1939. Échange de correspondance impliquant le sous-ministre de la santé, l'officier spécial de la division de l'Assistance publique, la sœur supérieure et le médecin de la crèche. 
niganais. Nous entendons montrer ici que les deux grands hôpitaux généraux tenus par des religieuses connurent des difficultés majeures à ce chapitre, et ce, malgré le soutien financier du gouvernement. Certes, ce dernier stimulait le processus de médicalisation de la société québécoise en facilitant l'hospitalisation pour une partie démunie de la population et en accélérant le développement des infrastructures. Mais la concurrence des établissements indépendants du clergé, de même que les réticences des médecins durant la crise des années 1930 devant la multiplication des admissions d'indigents allaient maintenir assez basses les populations de patients dans les deux grands hôpitaux généraux.

Tous les hôpitaux ne connaissaient pas des difficultés en ce qui a trait à l'approvisionnement en clientèle. Ainsi, au sanatorium Cooke, où la quasi-totalité des clients traités étaient accueillis sous le régime de l'Assistance publique, tous les lits (près de 170) étaient occupés presque en permanence. Dans son créneau, le sanatorium ne subissait pas de concurrence: aucun autre hôpital régional n'était aménagé pour recevoir des tuberculeux, si bien qu'avant son ouverture les tuberculeux étaient hospitalisés à l'Hôpital Laval de Québec. Disposant d'une large clientèle, financé essentiellement par l'État, c'était sans doute là le seul hôpital en Mauricie à l'abri des fluctuations de clientèle.

À l'Hôpital Saint-Joseph de Trois-Rivières et à l'Hôpital SainteThérèse de Shawinigan, la situation était tout autre. Malgré l'aide de l'État, les lits des deux grands hôpitaux généraux des deux villes n'étaient occupés qu'une partie de l'année. De 1916 à 1920 déjà, avant la Loi de l'Assistance publique, chaque lit de l'hôpital Saint-Joseph n'avait été utilisé qu'environ un jour sur deux, soit entre 150 et 188 jours par année. Il y eut bien une hausse de clientèle à partir de 1923 (figure 3), due en bonne partie à l'admission de bénéficiaires de l'Assistance, sans toutefois que la fréquentation de l'hôpital n'approche sa capacité d'accueil. La situation allait devenir critique durant la crise des années 1930, tant à Sainte-Thérèse qu'à SaintJoseph. En effet, la proportion de patients en mesure de payer les coûts d'une hospitalisation s'abaissa subitement, si bien que les revenus de ces deux hôpitaux allaient être constitués en bonne partie de l'argent fourni par l'Assistance publique. Témoin cette remarque trouvée dans les chroniques des Sœurs de la Providence de 1932-1933, à l'effet que leur établissement trifluvien subsistait «en grande partie par les revenus de l'Assistance publique ${ }^{61} »$. Témoin également toute

61. Chroniques des Sours de la Providence, 1932-1933, 68. 
FIGURE 3

\section{Admissions et jours d'hospitalisation à l'hôpital Saint-Joseph de Trois-Rivières de 1918 à 1941}

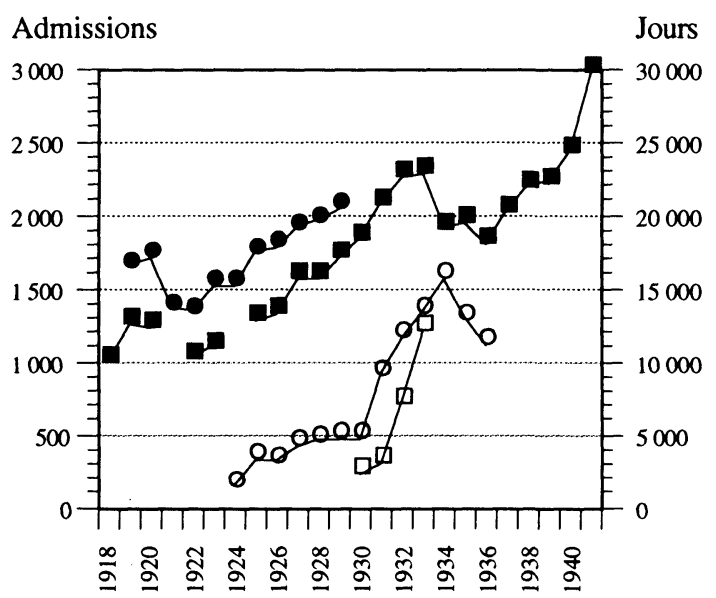

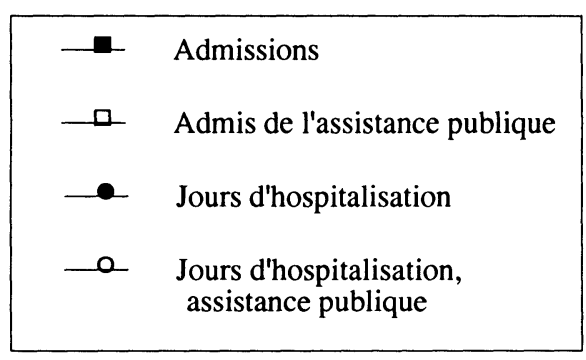

Sources: Chroniques des Soeurs de la Providence, Rapports annuels du SAP et Statistiques des institutions d'Assistance publique, années concernées.

une série de commentaires émis par la sœur responsable des chroniques à l'Hôpital Sainte-Thérèse de Shawinigan, signalant que les patients payants étaient rares, et qu'à certains moments la clientèle était formée essentiellement d'indigents bénéficiant de l'Assistance publique. En fait, de 1932 à 1936, ce fut le cas d'environ 50\% des clients admis ${ }^{62}$. La direction ressentit même le besoin de démontrer dans la presse «la fausseté de l'opinion répandue en certains milieux qu'il n'y a à Ste-Thérèse que des cas d'indigents ${ }^{63}$ ».

Divers facteurs doivent être évoqués pour expliquer la faible fréquentation des grands hôpitaux généraux tenus par les religieuses ${ }^{64}$ : une perception, dans la population, peut-être encore assez négative de l'hôpital, naguère lieu où les plus démunis allaient finir leurs jours ${ }^{65}$; coûts trop élevés de l'hospitalisation pour une bonne partie de la population qui, ne disposant que de bas revenus et sans assurance,

62. Rapports annuels de l'Hôpital Sainte-Thérèse publiés dans les journaux.

63. Coupure de journal non identifié, 1935, qui peut être consultée aux Archives des Sœurs Grises de la Croix d'Ottawa.

64. Il semble qu'à Montréal la situation était bien différente.

65. Un journaliste trifluvien note ainsi en 1917 un changement «dans la mentalité populaire qui se familiarise avec l'idée de l'hôpital». «L'Hôpital Saint-Joseph des TroisRivières», Le Bien Public, 22 février 1917, 1. 
hésitait vraisemblablement à prendre le chemin de l'hôpital. Nous nous pencherons ici plus particulièrement sur deux facteurs qui vinrent aggraver le problème de la non-fréquentation durant la crise: la concurrence des deux petits hôpitaux indépendants, et la décision des médecins de freiner l'admission des indigents.

Si les religieuses, en ces temps difficiles, avaient avantage à ce que les indigents soient admis nombreux sous le régime de l'Assistance publique, ce qui leur procurait un revenu, il n'en allait pas de même pour les médecins. En effet, les soins qu'ils dispensaient aux indigents étaient gratuits. Or, la clientèle payante étant moins nombreuse, leurs revenus baissaient au moment même où augmentaient les soins dispensés gratuitement aux indigents. Aussi décidèrent-ils, en guise de pression sur les pouvoirs publics et en vue d'obtenir une rémunération, de ne soigner que les cas d'urgence, ce qui eut pour effet d'endiguer le flot des admissions. Ainsi, alors que le nombre de patients admis annuellement à l'Hôpital Saint-Joseph, de 1102 en 1923, avait plus que doublé dix ans plus tard, il stagna durant quelques années avant de reprendre sa progression rapide (figure 3 ). Cette décision émanait de l'Association des médecins du district des TroisRivières et faisait suite à quelques années de mécontentement chez les médecins envers les modalités de l'aide sociosanitaire gouvernementale, comme le montre un article publié dans un journal local en 1932 par les médecins de l'Hôpital Saint-Joseph:

....avec la loi de l'Assistance Publique et le secours direct dans les circonstances actuelles, quelle est la classe de gens qui donne DIRECTEMENT le plus aux pauvres et aux indigents? L'épicier donne sa marchandise? Oui, mais contre remboursement par le secours direct. Il en est de même pour le boucher, pour le marchand et pour tous les autres fournisseurs, chacun pour ce qu'il donne est payé... et le prix du marché. Qu'est-ce que la loi de l'Assistance Publique donne aux médecins qui traitent les malades qui entrent dans les hôpitaux sous le couvert de cette loi? Qu'est-ce que les hôpitaux donnent aux médecins qui traitent les malades des dispensaires (...)? A ces deux questions, la réponse est la même: RIEN ${ }^{66}$.

Les médecins se résolurent à diminuer non seulement le nombre des admissions d'indigents aux soins internes, mais également aux soins externes délivrés dans les différents dispensaires que tenaient les hôpitaux. Dès 1933, les médecins rattachés à l'Hôpital Saint-Joseph exigèrent et obtinrent de la direction que seuls les indigents relevant

66. Le Nouvelliste, 29 octobre 1932, 3. 
de la société Saint-Vincent-de-Paul soient admis aux dispensaires, avec l'exception toutefois de ceux référés par les sœurs «visitatrices ${ }^{67} »$. Mais ils éliminèrent cette exception en 1935 par une résolution ayant pour but, selon leurs propres termes, «de venir en aide à la profession médicale, et de faire cesser l'exploitation dont elle est la victime $^{68}{ }^{\text {}}$. La forte baisse du nombre de nouveaux cas repérés durant la crise au dispensaire antivénérien de l'Hôpital Saint-Joseph, établi en 1921 dans le cadre du programme fédéral-provincial de lutte contre les maladies vénériennes, résulte vraisemblablement de leurs réticences: alors que de 1924 à 1931, entre 150 et 207 nouveaux cas furent rapportés annuellement (les données sont manquantes pour 1925, 1926, 1932-1933, 1935, 1937), leur nombre passa à 10 en 1934, 48 en 1936 et 45 en $1938^{69}$. Au dispensaire de Shawinigan ouvert vers 1932, il demeura également assez bas durant la crise, variant entre 16 et 32 de 1934 à 1939. Une baisse de l'intérêt des médecins pour le programme gouvernemental de lutte antivénérienne semble s'être aussi manifestée dans l'ensemble de la province, si l'on en juge par les commentaires du directeur de la Division des maladies vénériennes du Service provincial d'hygiène ${ }^{70}$.

Dans un contexte où le nombre de lits disponibles en région dépassait la demande, les difficultés des grands établissements à obtenir une fréquentation élevée étaient dues également à la présence des deux petits hôpitaux trifluvien et shawiniganais, qui comptaient respectivement 50 et 42 lits en $1931^{71}$. La concurrence pour la clientèle payante entre l'Hôpital Sainte-Thérèse (75 lits d'hôpital) et l'hôpital des compagnies à Shawinigan, renommé le Joyce Memorial en 1931 lors d'un agrandissement effectué l'année même où on érigeait l'Hôpital Sainte-Thérèse, l'illustre bien. Les chroniques des religieuses contiennent quelques remarques à ce sujet. Ainsi, lorsqu'en 1938 le directeur du Joyce Memorial vint s'enquérir des prix pratiqués par l'Hôpital Sainte-Thérèse et proposer une politique tarifaire commune, on lui fit remarquer que son établissement coupait depuis longtemps dans les prix «afin d'empêcher les gens de venir $\mathrm{ici}^{72}$ ».

67. Les sœurs «visitatrices» avaient pour tâche de venir en aide aux pauvres de TroisRivières. Elles amassaient des dons en passant de porte en porte, distribuaient des biens de toutes sortes, veillaient les malades, dirigeaient des indigents vers les dispensaires, etc.

68. Délibérations du bureau médical de l'Hôpital Saint-Joseph, 1935, 80.

69. Voir les rapports annuels de la Division des maladies vénériennes. Ils contiennent aussi des données sur le nombre de cas sous traitement, malheureusement inutilisables avant 1938 d'après le directeur de la Division dans le Premier rapport annuel du ministère de la Santé et du Bien-être social pour les années 1935 à 1941, 124.

70. Rapports annuels de la Division des maladies vénériennes pour les années 1935 et 1936, respectivement 75 et 80 .

71. Directory of Hospitals in Canada, 1931, 7-8.

72. Archives des Sœurs grises de la Croix d'Ottawa, Chroniques (novembre 1938): 72. 
Soulignons que l'hôpital Joyce Memorial parvenait à attirer sa clientèle en dépit des recommandations des membres du clergé à l'effet que leurs ouailles aillent se faire soigner à l'hôpital tenu par les religieuses. Ainsi, dans une lettre confidentielle envoyée en 1943 à la directrice de l'établissement, un curé d'une des paroisses de Shawinigan affirmait s'être fait un devoir depuis dix ans d'envoyer ses «gens le plus possible à l'Hôpital Ste-Thérèse en les détournant du 'Joyce ${ }^{73}$ ". Lorsqu'une pensionnaire des Ursulines soignée par un médecin rattaché à l'hôpital Joyce Memorial dut être hospitalisée en 1939, elles refusèrent catégoriquement que la malade y soit envoyée; aussi, dut-on exceptionnellement admettre le médecin de l'hôpital concurrent à venir opérer sa patiente à l'Hôpital Sainte-Thérèse ${ }^{74}$. Ce qu'il importe de noter ici, c'est que dans une ville dont pourtant environ 5 ou $6 \%$ seulement de la population n'était pas catholique, l'influence du clergé catholique ne suffit pas à briser la concurrence de l'hôpital des compagnies: bon nombre de patients payants, donc de ceux qui pouvaient choisir l'établissement où ils seraient soignés, allaient au Joyce Memorial.

\section{CONCLUSION}

Plusieurs intervenants ont influé sur le développement et l'organisation des services hospitaliers à Trois-Rivières et à Shawinigan de la fin du XIX ${ }^{\mathrm{e}}$ siècle à 1939 . Le clergé, bien sûr, propriétaire du plus grand hôpital régional et gérant des trois principaux établissements en terme de lits, joua un rôle de premier plan. Mais il dut compter avec l'influence grandissante des médecins, de même qu'avec le poids de la grande entreprise et de l'État. Les premiers, de plus en plus revendicateurs, se firent pressants en vue d'obtenir des équipements modernes, et exercèrent durant la crise un contrôle beaucoup plus serré qu'auparavant sur les admissions des indigents. Dans le cadre de la nouvelle médecine hospitalière qui se mettait en place, le clergé dépendait d'ailleurs des détenteurs du savoir médical, notamment en ce qui a trait à l'approvisionnement en patients payants devenus une source de financement non négligeable.

Durant les années 1910, malgré l'opposition du clergé, l'intervention de la grande entreprise permit l'établissement de petits hôpitaux laïques. Ce fut vraisemblablement la législation sur les accidents du travail qui incita les grandes entreprises mauriciennes à s'introduire de la sorte dans le champ des soins de santé. C'était donc

73. Archives des Sœurs grises de la Croix d'Ottawa, chemise 4B1, lettre du 19 février 1943 du curé C. Tremblay à la supérieure de l'établissement.

74. Archives des Sœurs grises de la Croix d'Ottawa, Chroniques, 25 mars 1939, 78. 
là, en partie tout au moins, un effet indirect d'une intervention de l'État. Mais l'influence de celui-ci sur le développement des services hospitaliers allait se faire beaucoup plus directe à partir des années 1920. Par l'application de la Loi de l'Assistance publique de 1921, l'État soutint en effet une hausse de la fréquentation des hôpitaux et finança l'extension et le renouvellement des infrastructures hospitalières, tout en se donnant des moyens de pression sur les autorités des établissements recourant aux subsides gouvernementaux.

Le système hospitalier régional (Trois-Rivières/Shawinigan/ Grand-Mère/La Tuque) qui prenait ainsi forme peut être divisé en deux grandes branches. La première était constituée d'établissements dispensant la majorité des soins aux indigents et recevant à ce titre l'aide de l'État, tous dirigés par des religieuses bien qu'elles n'étaient propriétaires que de deux sur quatre, les deux autres appartenant à une société philanthropique et à une municipalité. La seconde branche était formée de petits hôpitaux laïques qui prospéraient essentiellement sans l'aide de l'État grâce à une clientèle en mesure de payer son séjour. On y trouve un hôtel offrant une villégiature médicale et un hôpital général dirigés par des médecins propriétaires, de même que deux hôpitaux généraux appartenant à de grandes entreprises.

Les services hospitaliers en Mauricie ont connu des transformations profondes de la fin du XIX ${ }^{\mathrm{e}}$ siècle à 1939. À plus d'un titre, l'idée fréquemment exprimée - déjà contestée par quelques auteurs ${ }^{75}$ - selon laquelle le système hospitalier québécois de la première moitié du $\mathrm{XX}^{\mathrm{e}}$ siècle, hors des deux principaux centres urbains, était relativement homogène, plus ou moins stationnaire du point de vue de l'organisation et des rapports de pouvoir, dominé presque sans partage par le clergé et caractérisé par une quasi-absence de l'État ne concorde pas avec ce que nous avons observé en Mauricie. Enfin, les besoins spécifiques de la grande entreprise paraissent avoir joué un rôle méconnu à ce jour dans les transformations de l'hôpital au Québec, rôle qu'il conviendrait d'étudier plus en profondeur.

75. Normand Perron en particulier, op. cit. 